\title{
Pierre Larousse. Du Grand Dictionnaire au Petit Larousse, sous la direction de Jean Pruvost et de Micheline Guilpain-Giraud
}

\section{Hélène Giaufret}

\section{(2) OpenEdition \\ 1 Journals}

\section{Édition électronique}

URL : https://journals.openedition.org/studifrancesi/41397

DOI : $10.4000 /$ studifrancesi.41397

ISSN : 2421-5856

Éditeur

Rosenberg \& Sellier

\section{Édition imprimée}

Date de publication : 1 juillet 2004

Pagination : 234-236

ISSN : 0039-2944

\section{Référence électronique}

Hélène Giaufret, «Pierre Larousse. Du Grand Dictionnaire au Petit Larousse, sous la direction de Jean Pruvost et de Micheline Guilpain-Giraud », Studi Francesi [En ligne], 142 (XLVIII | I) | 2004, mis en ligne le 30 novembre 2015, consulté le 09 septembre 2021. URL : http://journals.openedition.org/ studifrancesi/41397; DOI : https://doi.org/10.4000/studifrancesi.41397

Ce document a été généré automatiquement le 9 septembre 2021.

\section{cc) $(9)$}

Studi Francesi è distribuita con Licenza Creative Commons Attribuzione - Non commerciale - Non opere derivate 4.0 Internazionale. 


\title{
Pierre Larousse. Du Grand Dictionnaire au Petit Larousse, sous la direction de Jean Pruvost et de Micheline Guilpain-Giraud
}

\author{
Hélène Giaufret
}

\section{RÉFÉRENCE}

Pierre Larousse. Du Grand Dictionnaire au Petit Larousse, sous la direction de Jean PRUVOST et de Micheline GUILPAIN-GIRAUD. Avec la collaboration de Julie DE BLOIS, Paris, Honoré Champion, 2002, pp. 368.

1 Ce volume recueille les contributions réunies lors du colloque international de Toucy, organisé par l'Association Pierre Larousse à l'occasion de la parution aux Editions Champion électronique d'un DVD-ROM du Grand Dictionnaire universel du XIXe siècle. Il permet une synthèse entre l' œuvre lexicographique de Pierre Larousse et les autres versants (lexicologie, grammaire et style) de sa multiforme activité.

Dans la Préface, Jean PRuvost après un tour d'horizon des études laroussiennes présente la structure du recueil dont la première partie est centrée sur les dictionnaires et les manuels de P. Larousse. Jean-Yves MolliER (L'œuvre de Pierre Larousse, pp. 37-47) présente l'œuvre du pédagogue, le caractère novateur de ses textes, manuels scolaires et ouvrages destinés aux maîtres. Le pédagogue se double chez lui d'un encyclopédiste, plus intéressé par la lexicographie que par la lexicologie, se situant dans la lignée de Diderot comme en témoigne l'élaboration du Grand dictionnaire universel du XIXe siècle (désormais GDU) qui lui permet de donner, outre les connaissances indispensables aux hommes de son temps, son avis sur «la politique, la morale, la philosophie, la religion, la littérature moderne» (p.42), souvent au détour 
inattendu d'un article. C'est que Larousse est un intellectuel engagé, républicain, pacifiste et libertaire.

3 J. PRUvost intitule sa contribution Du Nouveau Dictionnaire de la Langue française (1856) au Grand Dictionnaire universel du XIXe siècle (1866-1876). D'un monument à l'autre (pp.49-73). C'est autour de la métaphore du «monument» que se construit son article, métaphore omniprésente dans le GDU comme en témoigne l'article consacré à l'auteur lui-même, article fortement autobiographique (document rare et précieux) et donc particulièrement révélateur. Le triple définisseur générique est celui de "grammairien, lexicographe et littérateur». Les marques spécifiques se présentent selon le schéma chronologique des étapes de la production pour se terminer par le GDU, défini comme «une œuvre monumentale en son genre» (p. 54). Dans la Préface, P. Larousse utilisait déjà les métaphores de monument, édifice, statue, forteresse. Cette œuvre se définit comme œuvre nourricière, œuvre fondatrice, collective et collectrice.

Face à ce monument funéraire, le Nouveau Dictionnaire de la langue française (désormais NDLF) apparaît comme l'œuvre-berceau, qui aura dans sa filiation le Petit Larousse illustré, édité en 1905 et devenu une institution. Pourtant le premier dictionnaire n'est pas le brouillon de son devancier. L'œuvre de Larousse se place sous le signe de la dichotomie petit/grand dans une démarche non de réduction, comme c'est souvent le cas en lexicographie. Les points de contact entre les deux œuvres apparaissent comme les critères communs de "ces deux monuments» (p. 60): il s'agit de dictionnaires de langue, comme l'A. le souligne par la suite, affirmant que c'est par une méprise compréhensible que le public appréhenda le GDU comme un dictionnaire encyclopédique alors qu'il existe un équilibre entre les deux aspects et que, sur certains points («choix des exemples, découpage sémantique» (p. 64)) le traitement linguistique de Larousse est remarquable. Il est vrai qu'il s'agit aussi de dictionnaires encyclopédiques: deux des trois parties ajoutées au corps du NDLF relèvent de cette dimension. Par ailleurs ce sont des "dictionnaires fractionnés», ce sont des «dictionnaires militants, initiateurs et fondateurs». Les traits distinctifs qui les opposent concernent le public auquel ils sont respectivement destinés, la diffusion, millésimée et en un seul volume du premier et la publication échelonnée (1864-1876) de l'autre dont le texte ne sera plus modifié jusqu'en 1930. Le dernier critère est auctorial: le premier dictionnaire est une œuvre personnelle, le second s'élabore avec l'aide de collaborateurs. L'A., à travers la lecture des préfaces, montre que le NDLF a déjà en germe les éléments théoriques qui seront à la base du GDU: importance de la réflexion linguistique, distinction clarifiante entre dictionnaire de langue et dictionnaire encyclopédique, option raisonnée en faveur d'une description synchronique de la langue. Le NDLF, dictionnaire prototype, apparaît comme une ébauche du processus définitoire adopté dans le GDU, il représente une tentative réussie de sélection et de répartition de la nomenclature.

Elisabeth GRIMALDI (Interactions entre fonction encyclopédique et fonction linguistique: les réponses des «grands dictionnaires» du XIXe siècle, pp. 77-113) entend montrer comment circule la frontière entre dictionnaire de langue et dictionnaire encyclopédique et comment se répartissent les deux fonctions mises en œuvre. Après avoir rappelé les paramètres de classification, en particulier grâce à la lecture de l'article «Dictionnaire» de l'Encyclopédie, et signalé qu'au XIXe siècle, seule l'Académie résiste à la requête d'éléments informationnels de la part du public, elle affronte le problème des critères discriminants que l'on pourrait trouver dans l' «armature» de l'ouvrage (titre, préfaces, 
table des abréviations, tableaux de conjugaisons, listes de corrélats linguistiques, marques de domaine..) qui ne permettent pas de préjuger de la réalisation. La recherche s'étend à la nomenclature dont l'extension à noms propres, termes de lieux, termes scientifiques ou techniques est traditionnellement considérée comme critère discret, pour reconnaitre que la sélection s'opère selon des critères socio-historiques ou idéologiques «extralinguistiques plus qu'épilinguistiques» (p.90). A travers une analyse comparée de dictionnaires allant du Furetière au GDU en passant par l'Encyclopédie, l'A. montre que peu à peu s'instaure une disposition tranchée qui établit une frontière entre éléments linguistiques et encyclopédique sans que disparaisse la porosité même de cette limite, due à «l'ambiguïté inéluctable du sens» (p.110).

6 H.MitTeRAND dans Zola et Le Grand Larousse Universel du XIXe siècle de Pierre Larousse (pp. 115-125) illustre la corrélation entre les œuvres: incursions de Zola dans l'univers de Larousse, néo-encyclopédisme commun et rôle du GDU dans la genèse des RougonMacquart.

7 S.DELESALLE inaugure la partie consacrée aux grammaires Larousse par un article intitulé Les lexicologies et la lexicographie (pp.129-141). Elle y montre que les premiers travaux de Larousse (grammaires, méthodes, traités publiés entre 1849 et 1856) sont centrés sur le lexique et qu'une relation étroite unit la production lexicographique successive à ces élaborations grammaticales: Larousse y invite à «travailler la grammaire en se centrant sur les mots, analyser les formes par rapport au sens, commenter les choses en étudiant les signes» (p. 140).

8 E. S. KARABÉTIAN s'attache à analyser La conception du style chez Pierre Larousse: la parenté avec les arts d'écrire antiques (pp. 143-156). Il voit dans les ouvrages pédagogiques une réactualisation des conceptions grecques et latines avec leur composante rhétorique (importance donnée aux figures de mots), la place accordée à l'expression littéraire, le style conçu comme «art d'écrire»,

9 La deuxième partie est centrée sur les petits et grands Larousse. Elle s'ouvre sur un riche article de J. de Blois (Le Dictionnaire «Petit Larousse» d'hier à aujourd'hui: «Toujours le même et toujours différent» ou L'évolution d'un produit dictionnairique, pp. 161-204) qui retrace l'évolution de cette institution nationale que représente le Petit Larousse (PL) en ce qui concerne le titre, les refontes et les tirages, la présentation (reliure, éditions de luxe, version électronique), la couverture, le papier, la page de titres, les auteurs, le contenu, l'iconographie, la nomenclature, la devise et les logos, les millésimes, les lettrines et vignettes, la mise en page. Dans la fidélité à sa mission d'origine, le PL a su s'adapter: le tournant le plus apparent se situe à la fin des années 50 et l'évolution a connu une accélération à la fin des années 80 .

10 J.-Cl. BOULANGER situe Le Petit Larousse au Québec: brève histoire et influence (pp. 205-222). Ayant longtemps joui d'une situation de monopole, le PL a constitué un éminent instrument linguistique, pédagogique et un outil d'auto-apprentissage culturel. Ce n'est que tardivement (1959-1997) toutefois que le Québec s'introduit dans le PL. En 1968 sont répertoriés les premiers canadianismes dont la présence ira se multipliant en même temps que s'instaure une collaboration de la part de linguistes québécois et que le québécisme passe du statut de régionalisme à celui e variante francophone.

11 A. Lehmann retrace L'évolution culturelle du Petit Larousse: l'exemple de la sexualité (pp. 223-239). Domaine tabou à l'origine, le sexe est banni des premières éditions en raison de leur vocation prioritairement pédagogique. La censure ne sera levée que 
progressivement dans une évolution qui se termine en 1980. L'A. retrace l'évolution en ce qui concerne les mots et les acceptions relatives à l'appareil génital masculin et féminin . Les dates d'apparition font apparaître que la censure est plus sévère et le tabou plus fort sur l'appareil génital masculin et que les définitions évoluent de la rétention à l'accroissement de l'information.

F. CORBIN invite à considérer l'iconographie (Image-outil et image-spectacle: sur la dualité de l'iconographie du petit Larousse Illustré (Millésimes 2000 et 2001), pp. 241-287). La présence de l'iconographie obéit à deux impératifs: l'un utilitaire (fonctionnalité: l'image apporte de l'information), l'autre relevant de l'attractivité. Globalement l'iconographie apparaît comme un composant plutôt marginal lié à des items de nature encyclopédique. L'A. analyse l'articulation entre fonctionnalité et attractivité dans trois domaines, celui des images d'animaux et végétaux, de la haute technologie et des reproductions d'œuvres artistiques. Dans le premier cas, l'image participe à l'information sémanticoréférentielle selon un système de complémentarité avec l'article (traits saillants). En outre elle apporte des informations encyclopédiques. L'attractivité se manifeste par le choix de référents exotiques ou singuliers. Le domaine de la haute technologie connote de modernité le dictionnaire mais parfois l'iconographie se révèle inappropriée. L'insertion de reproduction d'œuvres artistiques, quantitativement plus nombreuses, révèle une certaine préciosité et relève parfois d'un élitisme culturel. L'article se termine par un examen des nouveautés introduites dans les millésimes 2001 et 2002 qui comptent 80 planches nouvelles contenues dans cinq cahiers et privées donc de toute fonction lexicographique mais témoignant de visées encyclopédiques et tendant à susciter l'émerveillement du lecteur devant la beauté et la diversité du monde.

G. Mоттет fait un bilan de la présence de Géographie et sciences de la terre dans les Dictionnaires Larousse: évolution de la pensée de 1964 à 1990 (pp.291-298). L'analyse de quelques entrées du Grand Larousse encyclopédique et du Grand Larousse en cinq volumes, révèle que grâce à la «précision rigoureuse des définitions et de la connaissance du moment» (p. 29) et aux représentations cartographiques, on peut reconstituer l'évolution de la science dans les deux domaines.

Le volume se clôt sur un article de G. TAVERDET sur Les régionalismes dans les dictionnaires (pp.301-313) qui souligne, à partir de quelques exemples, les lacunes et les inexactitudes dans la prise en compte et le traitement des variantes francophones (hélvétismes, belgicismes, québécismes, africanismes) aussi bien que des régionalismes du français hexagonal. 